\title{
Impacto del proceso de renovación urbana en la distribución interna de la población. Aproximación a su estudio a partir de la comparación del envejecimiento demográfico de Madrid entre 1986 y 1996
}

\author{
Ma José Aguilera Arilla y Mª Pilar González Yanci (U.N.E.D.)
}

RESUMEN: SUMMARY:

El envejecimiento demográfico del municipio de Madrid comienza a hacerse patente desde que en 1980 se superó la cifra del 10\% de ancianos en el conjunto de la población. Los Padrones Municipales de 1986 y 1996 evidencian que el proceso continúa y se acrecienta, siendo cada vez mayor el número de barrios envejecidos, fenómeno que ya no sólo se circunscribe a los centrales, sino que también afecta a los exteriores. Determinadas actuaciones, que suponen cambios en los usos del suelo, comienzan a producir un cierto rejuvenecimiento de áreas del interior, que anticipan posibles cambios futuros.
The demographic ageing of the city of Madrid has become an obvious matter since 1980, when the total amount of elderly people surpassed the $10 \%$ of the total population. The Official Registries of 1986 and 1996 come to prove that such process is not only still ongoing but also shows that there is an increasing number of ageing urban areas, which does not remain a phenomenon of the inner neighbourhoods but also extends to the suburban areas of the city. However, certain actions that imply changes in the utilisation of the ground are beginning to produce some rejuvenation of the inner areas, fact that anticipates potential future changes.

\section{INTRODUCCIÓN}

El último cuarto del siglo XX se caracteriza, en España, desde un punto de vista demográfico, entre otras cosas, por un acusado proceso de envejecimiento de la población. Madrid, que en el conjunto del país representa una isla relativamente joven, en un centro más envejecido, tam- 
bién sufre este proceso, considerada en sí misma, presentando un grado notable de envejecimiento que, además, se manifiesta con considerables diferencias espaciales, tanto a escala provincial como municipal, que ya ha quedado puesto de manifiesto en numerosos trabajos (Abellán, 1976; Aguilera, G. Yanci, 1989; García, 1989; López, 1992 y Rojo, Rodríguez y Fernández, 1993).

A través de un estudio que realizamos en 1987, con los datos del $\mathrm{Pa}$ drón Municipal de 1986, comprobamos la existencia de un claro contraste en la distribución espacial de los ancianos, cuya proporción disminuía conforme aumentaba la distancia al centro histórico, en consonancia con la propia evolución urbana. Desde aquella fecha ha transcurrido un período de tiempo suficientemente largo como para analizar la evolución que está sufriendo el proceso de envejecimiento. La existencia de un nuevo Padrón Municipal nos permite comparar y analizar los cambios producidos entre ambas fechas.

El primer cambio detectado no se refiere a la población, como podría esperarse, sino a la división administrativa municipal. En 1986 Madrid estaba dividida en 18 distritos y 120 barrios. Poco después, en 1988, se hizo una redistribución en 21 distritos que comprenden 128 barrios, modificándose algunos distritos del interior (como Arganzuela) y, sobre todo, los más exteriores del Este, sin que cambiara la extensión del término municipal. Este hecho supuso una dificultad inicial, que resolvimos adaptando todos los datos de 1986 a la nueva distribución, utilizando los datos de secciones censales que se incluían en la antigua y nueva división, con lo cual disponemos de tablas reelaboradas y, sobre todo, de unos mapas con la misma base, que permiten realizar la comparación entre las dos fechas y predecir lo que puede ocurrir en el futuro, siempre que no se produzcan nuevos cambios (en un caso, el barrio de Horcajo, carece de datos y en otros dos faltan algunos indicadores, como natalidad, mortalidad, etc. como puede apreciarse en los mapas.)

El objetivo perseguido con este trabajo es descubrir las tendencias que sigue el envejecimiento de los barrios y distritos de Madrid, a la espera de los resultados del Censo, que se llevará a cabo en el año 2001 y comprobar si en este proceso se aprecia ya alguna incidencia de las transformaciones internas resultantes de la dinámica urbana.

La hipótesis de la que partimos es que en la ciudad se producen determinados hechos que pueden tener repercusión en la distribución espacial de la estructura por edad de la población. Entre aquéllos se pueden 
citar algunos, que en los últimos años cobran cierta relevancia y son relativamente nuevos. Por ejemplo, la llegada de inmigrantes jóvenes al centro histórico, que puede detectarse incluso a simple vista; la rehabilitación de viviendas y edificios del casco antiguo, que estaba sufriendo un deterioro muy grande y, sobre todo, los cambios en los usos del suelo, que resultan de la transformación de grandes espacios, que pasan de su antiguo uso a uno nuevo, predominantemente residencial. En el caso de Madrid destaca la llamada operación "Pasillo Verde", mediante la cual, una amplia zona de la ciudad dedicada a estaciones, vías y almacenes ferroviarios, e industrias anejas, se está transformando en zona de viviendas. Otro tanto sucede con espacios que estaban ocupados por instalaciones militares, deportivas, cárceles, e industrias, que están pasando a ser de uso residencial. A lo que hay que añadir la transformación de espacios rurales periféricos en nuevos barrios de viviendas, conforme a los recientes planes urbanísticos (Valdebernardo, Las Rosas, Plan Chamartín... )

\section{COMPARACIÓN DE LA DISTRIBUCIÓN ESPACIAL DEL ENVEJECIMIENTO ENTRE 1986 Y 1996.}

Una vez adaptadas las cifras a la nueva delimitación administrativa, para realizar la comparación entre las dos fechas, se han cartografiado los resultados de algunos indicadores del envejecimiento, que resultan por sí mismos muy expresivos de la transformación habida.

El índice más sencillo es el de la proporción de ancianos en el conjunto de la población. En 1996 la proporción de ancianos en el total era de $17.9 \%$ habiéndose producido una diferencia de $5 \%$ con respecto a 1986 , en que aquélla era del $12.9 \%$. Considerado en cifras absolutas aún resulta más llamativo el incremento del número de ancianos, ya que, habiéndose producido una disminución del total de la población madrileña en 191.302 personas, en cambio los ancianos aumentaron su número en 118.964 con relación a los de 1986, con todas las repercusiones sociales que esto representa (como necesidad de residencias de ancianos, atención hospitalaria y sanitaria, adecuación de viviendas y equipamientos urbanos para las discapacidades, incremento de zonas verdes, lugares de ocio y recreo, necesidad de personas para atender a los mayores, etc.).

Si aceptamos con Paillat que el porcentaje del $20 \%$ marca el límite para considerar a una población envejecida, encontramos que en 1986 había veinte barrios por encima de este valor, mientras que en 1996 eran cincuenta y uno los que superaban tal cifra. Entre 1986 y 1996 hay un no- 
table incremento de ancianos en total y, además, es espectacular el aumento del número de barrios que pasan a la categoría de envejecidos, lo que supone una expansión del fenómeno, antes mucho más concentrado en los barrios más antiguos de la ciudad, coincidentes con lo edificado hasta el siglo XIX. En la primera fecha, los barrios con mayor proporción de ancianos eran los del distrito Centro (Palacio, Embajadores, Cortes, Justicia, Universidad y Sol) que corresponden a la más antigua superficie edificada de Madrid hasta la época citada. Con valores similares, por encima del $20 \%$, estaban los barrios de Recoletos, Goya y Lista, seguidos de Argüelles, Gaztambide, Arapiles, Almagro, Castellana, Ibiza y Moguer, todos pertenecientes al Ensanche de 1860. Fuera de éste, tan sólo era equiparable el barrio de Ríos Rosas, muy próximo y similar al Ensanche y los de Bellas Vistas, Valdemarín y El Goloso, con valores de 19.29, 19.67 y $54.48 \%$ respectivamente, en los que confluyen determinadas circunstancias, que ayudan a explicarlo (antiguo poblamiento de la que fue carretera de Francia en los dos primeros y presencia de una gran residencia pública de ancianos, en el último).

En la segunda fecha todo el distrito Centro supera ya el $24 \%$, con un máximo del $26 \%$ en Embajadores. Los distritos de la llamada "Almendra Central" están envejecidos en conjunto, por encima del $20 \%$, a excepción de los barrios de Retiro y Chamartín, que con el $19 \%$ resultan algo más jóvenes, debido a la incidencia que en ellos tienen las zonas más exteriores, en las que se estaba consolidando la edificación en torno a la arteria de circunvalación $\mathrm{M}-30$, que marca el límite de aquella zona. Fuera de este perímetro, en 1996, ningún distrito supera el $20 \%$, pero en varios de ellos ya aparecen barrios que sí lo hacen. Son los de El Pardo, Valverde, El Goloso, Casa de Campo, Argüelles, Puerta del Angel, Comillas, Opañel, Almendrales, Moscardó, San Diego, Ventas, Quintana, La Concepción, San Cristóbal, Simancas, Hellín, Amposta, Arcos y Rejas, todos situados inmediatamente a continuación de la Almendra Central y, en algún caso, más alejados, pero en estrecha relación con los principales ejes radiales de carreteras, de muy antigua edificación. (Nótese la excepción ya conocida de El Goloso, en que la citada existencia de una gran residencia de ancianos, en un pequeño volumen de población distorsiona la realidad). (Mapas 1 y 2 ).

Si en lugar de considerar a los mayores de 65 años, tomamos el límite de 75 años, cada vez más normal, dado el aumento de la esperanza de vida, aún resulta más evidente el envejecimiento de la población madrileña. En 1986 tan sólo 12 barrios tenían más del 10\% de su población por encima de los 75 años, mientras que en 1996 eran ya 27 los barrios que 


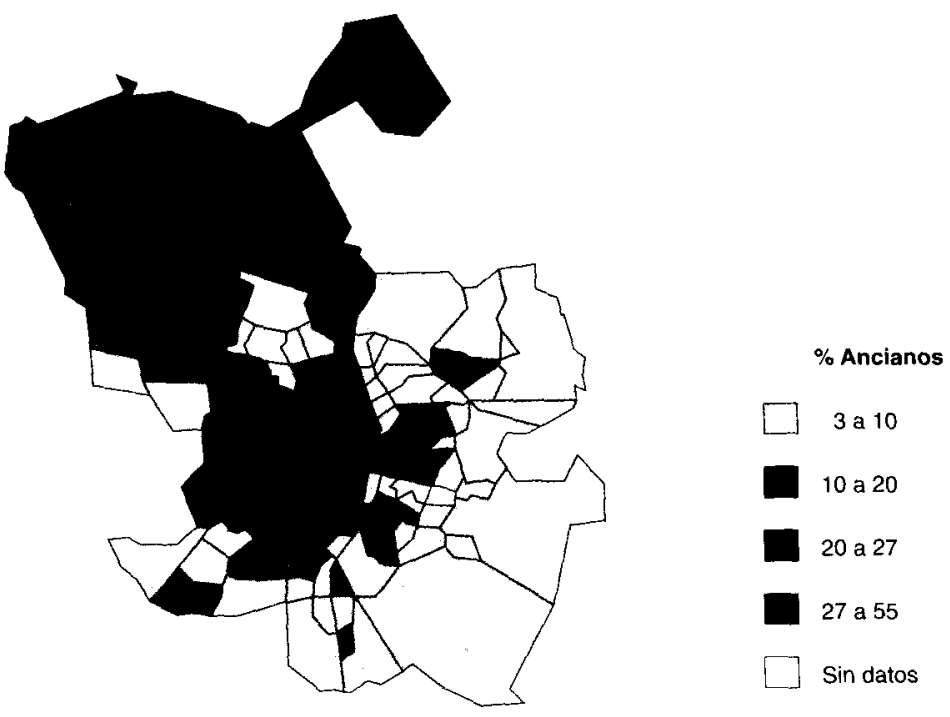

Mapa 1. \% de ancianos (> 65 años) en el total de la población) Distribución por barrios 1986

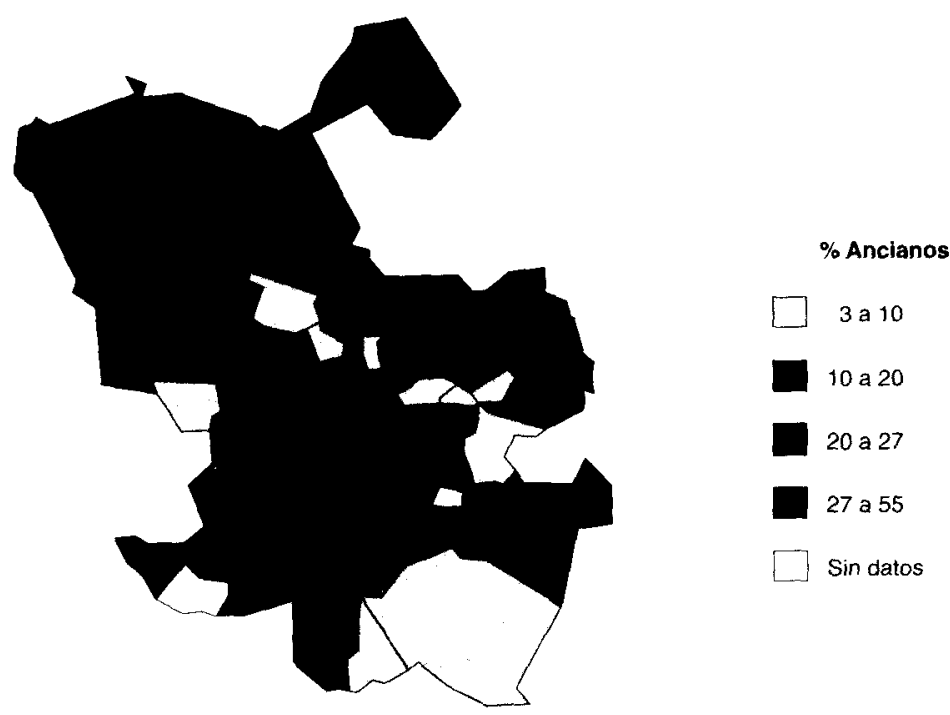

Mapa 2. \% de ancianos (> 65 años) en el total de la población) Distribución por barrios 1996 
superaban tal cifra. Lo que demuestra que, además de ser más los ancianos en relación con la población, hecho que también se ve influido por el descenso de la fecundidad, son más numerosos los que llegan a vivir más años, siendo, por tanto, más longevos (Mapas 3 y 4).

El índice de vejez, consistente en establecer la relación entre los mayores de 65 años y los menores de 15 (edades de comienzo y final de la vida laboral) nos aporta más información respecto al envejecimiento real, poniendo de manifiesto, en cierto modo, la influencia que tiene en el proceso el descenso de la fecundidad, que en el municipio de Madrid se presenta, en general, muy baja, pero con contrastes espaciales por barrios muy marcados (vid mapa 7 que muestra la tasa de natalidad por barrios en 1995).

El valor de 0.5 marca el límite entre sociedad envejecida y joven. Teniendo en cuenta este valor, se puede apreciar el cambio entre las fechas consideradas. En 1986 había 34 barrios por debajo del valor 0.5, mientras que en 1996 eran tan sólo 13 los que estaban en esta situación. En el primer caso se trata de los barrios de: El Plantío, Aravaca, Fuente la Reina, Peñagrande, El Pilar, La Paz, Valdezarza, Mirasierra, Valdefuentes, Costillares, Atalaya, Colina, San Juan Bautista, San Pascual, Salvador, Canillas, Pinar de Rey, Apóstol Santiago, Palomas, Canillejas, Alameda de Osuna, Aeropuerto, Timón, Rosas, Pueblo Nuevo, Arcos, Ambroz, Casco Histórico de Vicálvaro, Pavones, Vinateros, Marroquina, Media Legua, Portazgo, Palomeras SE, Santa Eugenia, Casco Histórico de Vallecas, Entrevías, San Fermín, Los Rosales, Butarque, San Andrés, Los Ángeles, Orcasitas, Buenavista, Aluche, Las Águilas y Campamento, todos ellos situados en los distritos más exteriores, fuera de la Almendra Central. No obstante, en el interior de ésta, aunque bastante periféricos, aún había tres barrios jóvenes, según este índice (Adelfas, Estrella y Legazpi). En la fecha de 1996 ya sólo están por debajo del 0.5 los barrios de: Aravaca, Mirasierra, La Paz, Costillares, Valdefuentes, Piovera, Palomas, Alameda de Osuna, Rosas, Casco histórico de Vallecas y Butarque, en la periferia, y un único barrio en la Almendra Central, el de Legazpi.

La comparación de los mapas 1 y 2 con los mapas 5 y 6 refleja claramente las afirmaciones anteriores, que muestran que no sólo es grande el envejecimiento y que va en aumento, sino que, a futuro, va a ser más preocupante la situación, por ser los jóvenes especialmente poco numerosos.

En relación con el envejecimiento resulta también expresivo analizar la diferenciación espacial del comportamiento demográfico de la población. Los índices de natalidad y mortalidad de 1995 indican que hay nota- 
Impacto del proceso de renovación urbana en la distribución interna de la población...

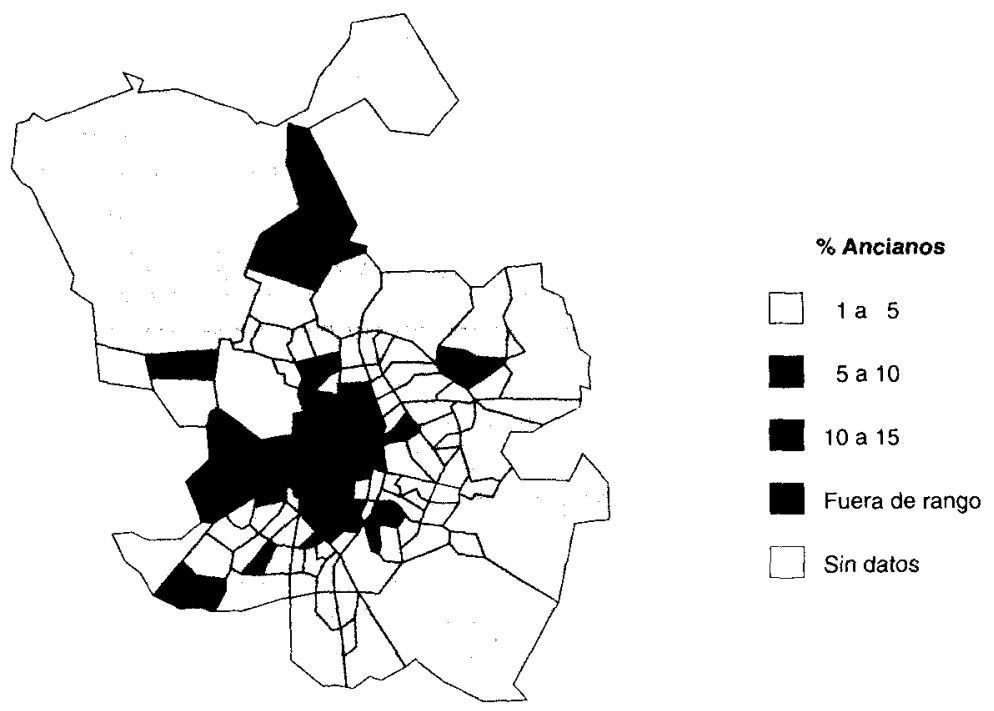

Mapa 3. \% de ancianos (> 75 años) en el total de la población) Distribución por barrios 1986

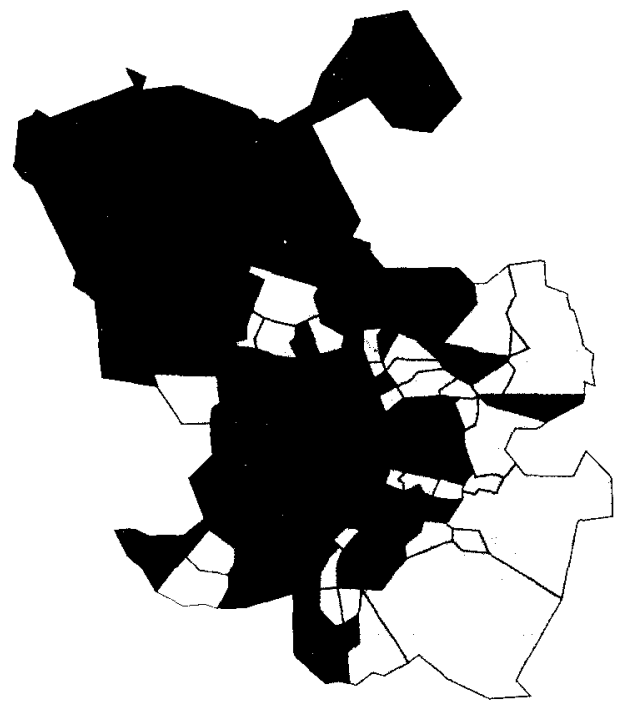

\% Ancianos

[ 1 a 5

5 a 10

10 a 15

Fuera de rango

Sin datos

Mapa 4. \% de ancianos (> 75 años) en el total de la población) Distribución por barrios 1996 
bles diferencias por barrios. Mientras que las tasas de mortalidad se relacionan bastante bien con la distribución espacial del envejecimiento (siendo más elevadas allí donde hay más ancianos), las de natalidad, que también muestran diferencias espaciales, se presentan más uniformemente bajas, sin la relación previsible (a mayor número de viejos menor natalidad) con la estructura demográfica. Esto indica que hay un marcado descenso de la natalidad en general, que hará, que a futuro, sea aún más fuerte el envejecimiento de los barrios, tanto los internos como los periféricos, corroborando lo que se evidenciaba con la observación del indice de vejez (Mapas 7 y 8 ).

El análisis del mapa del crecimiento de la población del periodo 8696 , muestra también que en él hay un gran contraste espacial. El centro y los barrios periféricos más antiguos y consolidados, pierden población. En cambio, los barrios de nueva edificación, situados en el exterior, sobre todo los de la zona Este, son los que manifiestan crecimientos positivos, incluso destacables. En este hecho subyace la influencia, no tanto de la fecundidad elevada, que no se da en Madrid, como la de la afluencia de nuevos habitantes, llegados a los barrios donde se construyen nuevas viviendas. De ellos, unos son inmigrantes, de procedencia extraprovincial o incluso extranjera, mientras que otros son, en buena medida, jóvenes que se desplazan desde sus barrios de nacimiento, al emanciparse. Además, en los barrios más antiguos, que eran desde 1986 los más envejecidos, la pérdida de población produce una cierta ralentización del proceso de envejecimiento, que es proporcionalmente menor al que se está produciendo en los barrios circundantes (Mapa 9).

\section{IMPACTO DEL PROCESO DE RENOVACIÓN URBANA EN LA DISTRIBUCIÓN INTERNA DEL ENVEJECIMIENTO.}

En el progresivo envejecimiento, que como se ha visto va afectando a todos los barrios del centro hacia la periferia, se puede apreciar que el valor de la razón de la progresión no es igual en todos los casos. Muchos son los factores que influyen en tales diferencias. Por ejemplo, los barrios que ya estaban más envejecidos en 1986, del distrito Centro, envejecen en menor proporción que los del Ensanche, por diversas causas, como la pérdida de población por mortalidad, lógica al tratarse de una población inicial de más edad y por la terciarización de la actividad, que, aunque lleva muchos años produciéndose, continúa en el presente y también porque, en el sentido contrario, ganan población joven, tanto por renovación 


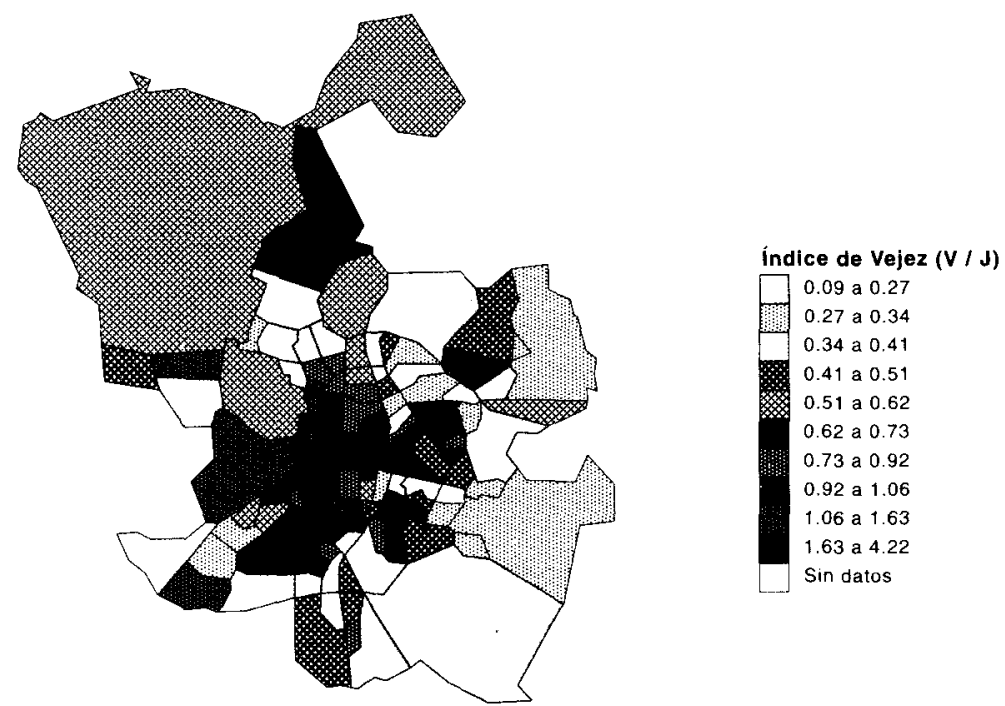

Mapa 5. Distribución por barrios del indice de vejez en 1986
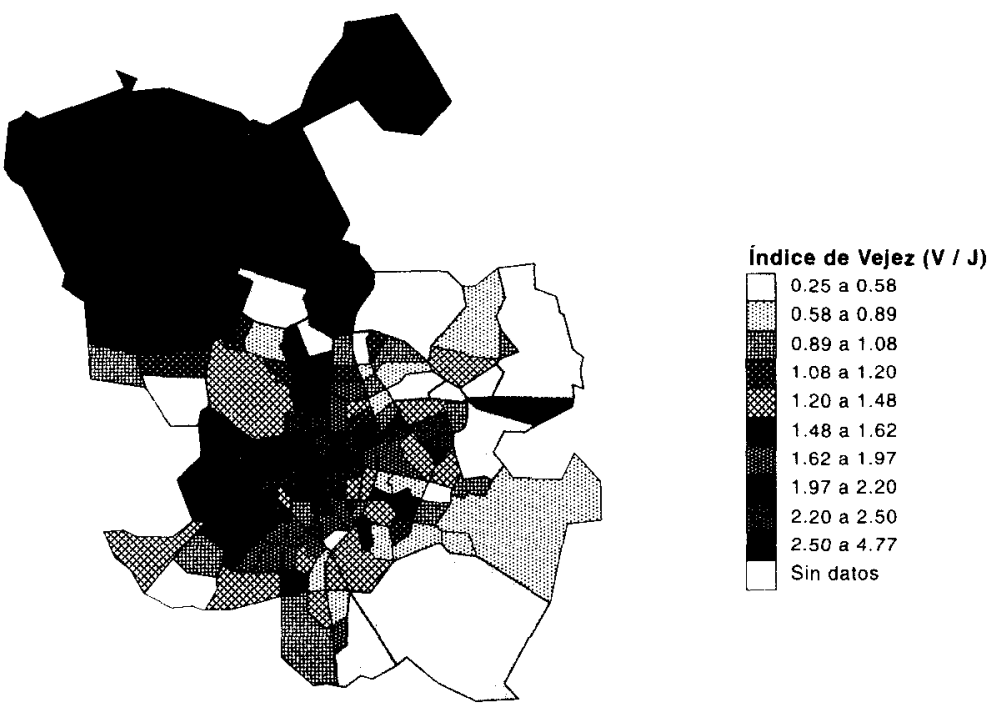

Mapa 6. Distribución por barrios del índice de vejez en 1996 


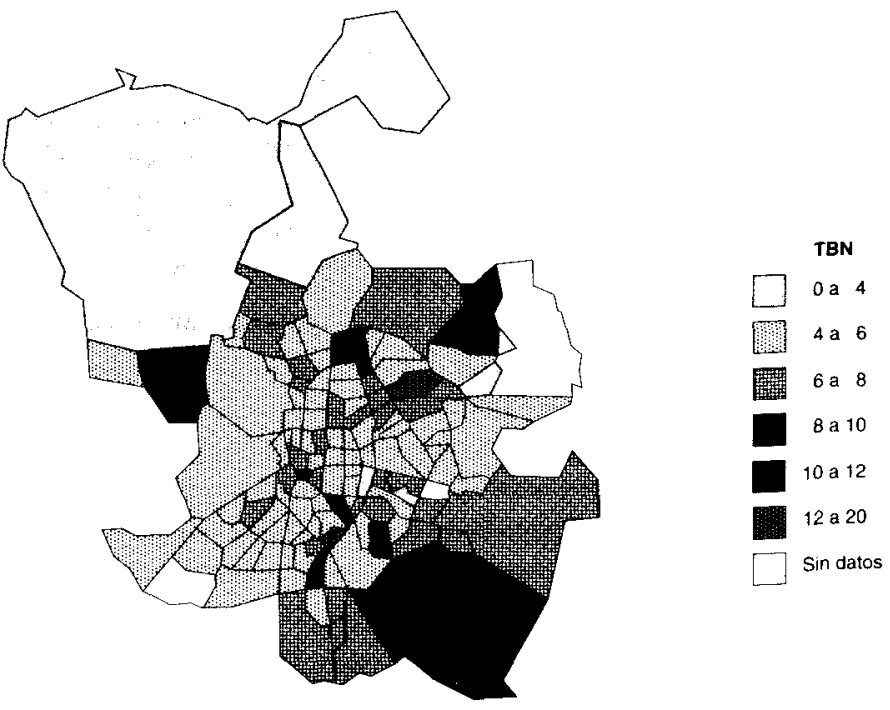

Mapa 7. Distribución por barrios de la tasa bruta de natalidad 1995

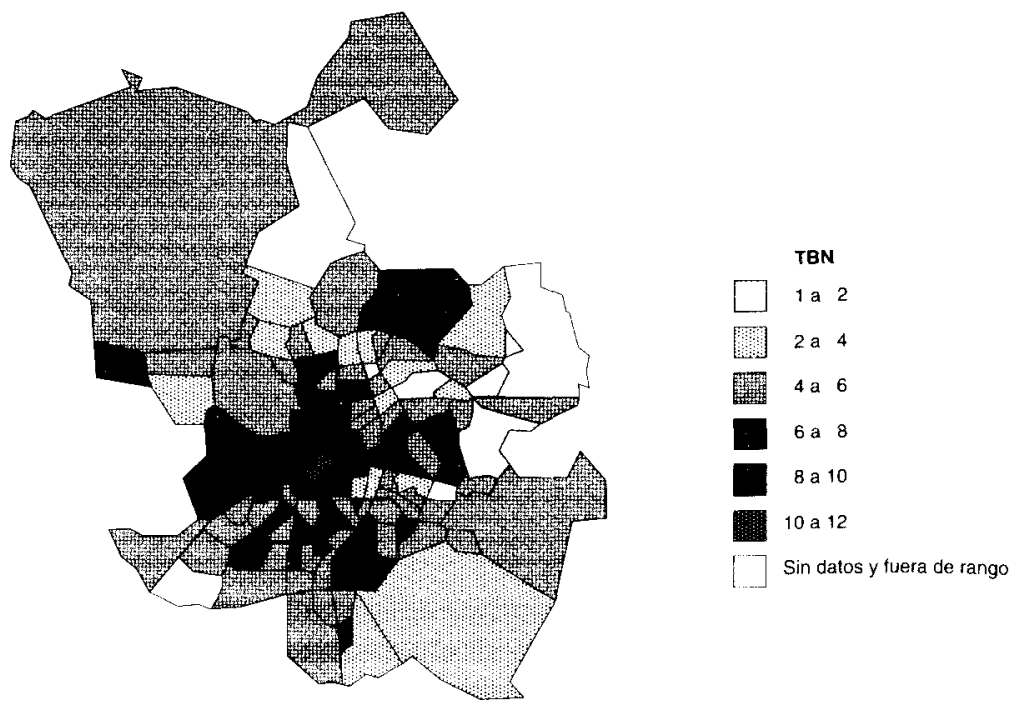

Mapa 8. Distribución por barrios de la tasa bruta de mortalidad1995 
Impacto del proceso de renovación urbana en la distribución interna de la población...

y rehabilitación del parque inmobiliario, como por la llegada de inmigrantes a viviendas de bajo nivel, abundantes en los barrios centrales, con la consiguiente modificación del índice de vejez.

Precisamente en este trabajo queremos llamar la atención sobre cómo puede ya apreciarse el impacto que producen las actuaciones de renovación y remodelación urbana en la distribución de la estructura por edad de la población, con el ejemplo del envejecimiento. No podemos considerar la remodelación urbana en conjunto que excede nuestros objetivos y merecería un trabajo aparte. Veremos, en cambio, algún ejemplo concreto.

Resulta paradigmático el caso de la operación urbanística denominada "Pasillo Verde" y zonas adyacentes, relacionadas con el cambio de uso del suelo, de lo que constituyó una zona industrial y ferroviaria, surgida en el Madrid del siglo XIX. El análisis de los datos de envejecimiento de los barrios por los que transcurre el citado Pasillo Verde muestra, entre 1986 y 1996, el mismo proceso de envejecimiento que la mayor parte del interior, sin que aún se observen cambios notables, que, sin duda, aparecerán tras el Censo próximo del año 2001, que en su momento estudiaremos. No obstante, ya se aprecia algún ejemplo como anticipo a esta situación. Nos centramos en un pequeño barrio de la zona próxima al Pasillo Verde, en la periferia de la Almendra Central, durante mucho tiempo zona industrial de Madrid, desde la implantación de las líneas ferroviarias en esta parte de la ciudad. Se trata del barrio de Legazpi, del que se está desplazando la actividad industrial y ferroviaria, ya casi inexistente y en el que se está pasando a un nuevo uso del suelo, ahora de carácter predominantemente residencial. Entre 1986 y 1996 este barrio es, junto al de Acacias, de carácter parecido, el único que manifiesta aumento de población y, sobre todo, posee la mayor tasa de natalidad de todo el distrito, el 17.8 por mil, junto a la menor tasa de mortalidad, el 5.9 por mil. Resulta un magnífico ejemplo de cómo un barrio interior, consolidado en el sigio XIX, del que cabría esperar un comportamiento similar al de los barrios de su entorno, puede mostrar una estructura demográfica y una evolución similar a la de los modernos barrios que van surgiendo en la periferia urbana, por un proceso de remodelación y cambio de uso del suelo, resultante de la planificación urbana en los centros de las ciudades. En otros barrios, en los que todavía no se nota esta desaceleración del envejecimiento, subyace el hecho de que las nuevas viviendas construidas alcanzan precios elevados, por el alto valor del suelo en las zonas centrales de la ciudad, lo que lo hace inasequible para los jóvenes, menores de treinta años, que suelen desplazarse a los municipios del Área Metropolitana. Los nuevos habitantes de la zona del Pasillo Verde son, en 


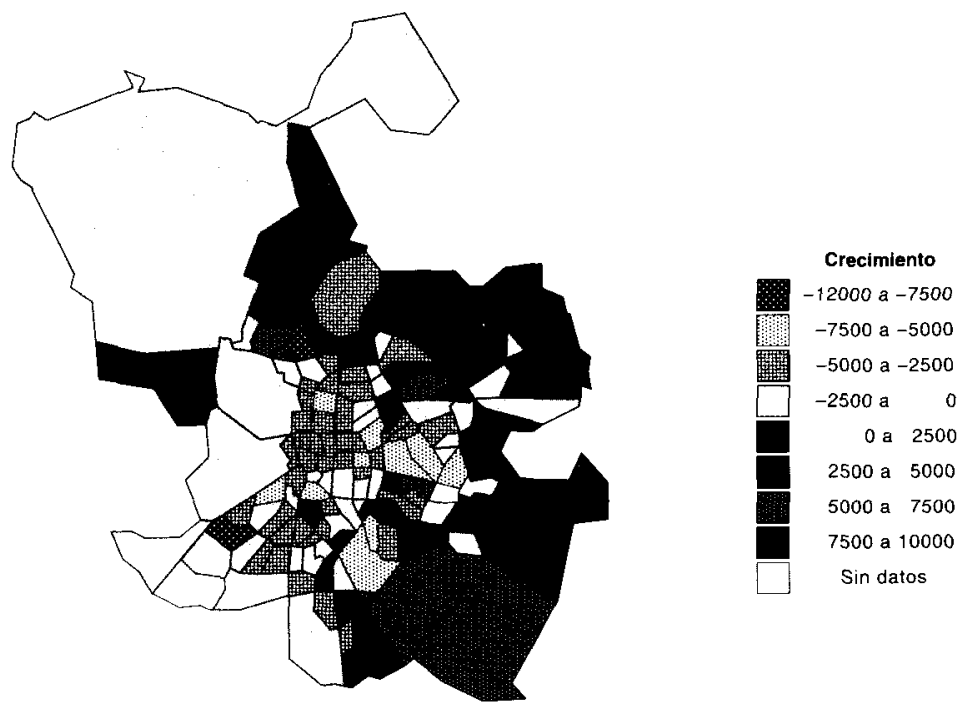

Mapa 9. Crecimiento de la población por barrios (1986-1996)

general, adultos, con o sin hijos, pero si los tienen con edades superiores a los quince años, por lo que, de momento, no se reflejan en los indicadores que acabamos de analizar. Es de esperar que tengan una repercusión en el próximo Censo del año 2001.

\section{CONCLUSIONES}

El envejecimiento de los barrios de las ciudades españolas sigue una progresión inversamente proporcional a la distancia al centro de la misma, siguiendo la evolución temporal del crecimiento urbano (González Yanci, M.P.. y Aguilera Arilla, M.J. 1989 ). Es decir, los barrios antiguos tienen población anciana en mayor proporción que los barrios nuevos. Esta progresión se puede ver interrumpida por acciones urbanísticas llevadas a cabo en el interior de la ciudad. De hecho, la rehabilitación y remodelación de los cascos históricos y los cambios de uso consiguientes (de industrial, ferroviario, militar, a residencial) dan lugar a áreas de rejuvenecimiento en determinados barrios interiores, fáciles de constatar cuando se trata de promociones grandes que generan un gran número de viviendas, frente a los casos aislados. A pesar de lo cual, la verdadera di- 
mensión del rejuvenecimiento no se pone de manifiesto por las excesivamente bajas tasas de natalidad y fecundidad que suele tener la población que alli se traslada. También en la actualidad la llegada de inmigrantes de países en desarrollo a zonas deterioradas de los cascos antiguos, en este caso con comportamiento demográfico de alta natalidad, produce un similar efecto de rejuvenecimiento.

En el caso de Madrid, donde se está llevando a cabo un importante proceso de renovación y expansión urbana, aparecen ya indicios de que en el proceso general de envejecimiento hay algunos barrios donde se produce un rejuvenecimiento que hace presagiar cambios en el futuro.

\section{BIBLIOGRAFIA}

ABELLÁN, A. (1976) "Estructura por sexo y edad de los distritos de Madrid" Estudios Geográficos. № 144, p. 303-317.

ABELLÁN, A. (Coordinador) (1996) Envejecer en España. Fundación Caja de Madrid, Madrid, $74 \mathrm{p}$.

AGUILERA ARILLA, M.J. y GONZÁLEZ YANCI, M.P. (1989) "Conirastes del envejecimiento demográfico entre el Municipio de Madrid y su Comunidad Autónoma". II Jornadas sobre Población Española. Palma de Mallorca, p. 69-79.

ANUARIOS ESTADÍSTICOS (Varios años) Ayuntamiento de Madrid.

AYUNTAMIENTO DE MADRID (1989) 21 Distritos. Madrid. Área de Coordinación y Participación del Ayuntamiento de Madrid. $351 \mathrm{p}$.

GARCÍA BALLESTEROS, A. et al. (1989) "El envejecimiento actual de la población madrileña" II Jornadas sobre Población Española. Palma de Mallorca, p. 207-227.

GAYMU, J. (1987) "Le vieillissement démographique des quartiers des grandes villes françaises" Espace Populations Sociétès, 1, p.125-135.

GONZÁLEZ YANCI, M..P. y AGUILERA ARILLA, M.J. (1989) "La diferenciación espacial del envejecimiento demográfico de los núcleos urbanos del País Vasco ". Espacio, Tiempo y Forma, serie VI nº 2, Geografía, UNED, Madrid, p. 191-220.

GONZÁLEZ YANCI, M.P. y AGUILERA ARILLA, M.J. (1989) "La diferenciación espacial del envejecimiento demográfico en los núcleos urbanos españoles." Actas de las II Jornadas sobre Población Española. Universitat de les Illes Balears. Palma de Mallorca, p. 249-62

LÓPEZ JIMÉNEZ, J.J. (1989) "El proceso de envejecimiento en la Comunidad Autónoma de Madrid" II Jornadas sobre Población Española. Palma de Mallorca, p. 273-284.

LÓPEZ JIMÉNEZ, J.J. (1992) Las personas ancianas en el Municipio de Madrid. Documento de Trabajo $n^{\circ} 41$. Ayuntamiento de Madrid, $290 \mathrm{p}$. 
ROJO PEREZ, F. RODRÍGUEZ RODRÍGUEZ, V. y FERNÁNDEZ MAYORALAS, G. (1993) "Concentración demográfica y geográfica de la población anciana en Madrid". Nuevos procesos territoriales XIII Congreso Nacional de Geografía. Sevilla, p. 527-532.

VINUESA ANGULO, J. (1990) Proceso de envejecimiento de la población de Madrid. Documento de Trabajo $n^{9} 8$. C.S.I.C. $37 \mathrm{p}$. 